\title{
CURVATURE AND COMPLEX ANALYSIS. III
}

\author{
BY R. E. GREENE AND H. WU ${ }^{1}$ \\ Communicated by S. S. Chern, October 30, 1972
}

This announcement is a sequel to Greene-Wu [1], [2]. Here we shall concentrate on Kähler manifolds of nonnegative curvature. Our first result improves Theorem 3 of [2], but the latter is needed in the proof of the former.

THEOREM 1. Let $M$ be a complete Kähler manifold with positive Ricci curvature and nonnegativesectional curvature. Let $K$ be the canonical bundle of $M$ and let $L$ be a holomorphic line bundle on $M$ such that $L \otimes K^{*}>0$ ( $K^{*}$ denotes the dual of $K ; L \otimes K^{*}>0$ means that the line bundle $L \otimes K^{*}$ possesses a Hermitian metric of positive curvature). Then $H^{p}(M, \mathcal{O}(L))=0$ for $p \geqq 1$.

The next theorem is the noncompact analogue of Kodaira's embedding theorem [4]. Its proof depends on Theorem 1 and is similar to Kodaira's proof in broad outline, but there are technical complications because of the noncompactness.

THEOREM 2. Let $M$ be a complete Kähler manifold with positive Ricci curvature and nonnegative sectional curvature. Then $M$ possesses nonconstant meromorphic functions. Specifically, given any compact set $K \subseteq M$, there exists a positive integer $N$ and a meromorphic mapping (see Remmert [5]) $\varphi: M \rightarrow P_{N} C$ such that $\varphi \mid K$ is a holomorphic embedding.

In [2], we conjectured that every complete noncompact Kähler manifold with positive sectional curvature must be a Stein manifold. The next theorem includes the solution of this conjecture as a special case. Recall that a subset $S$ of a Riemannian manifold is convex if, for any $p, q \in S$, at least one minimizing geodesic joining $p$ and $q$ lies in $S$.

THEOREM 3. Let $M$ be a complete Kähler manifold with positive Ricci curvature and nonnegative sectional curvature, and suppose that the canonical bundle of $M$ is topologically trivial. Then every convex open subset of $M$ is a Stein manifold.

The fact that any open convex subset of such a manifold $M$ is necessarily a Stein manifold should be compared with Theorem 7 of [1]; of course the

\footnotetext{
AMS (MOS) subject classifications (1970). Primary 53C55, 32C10, 32E10; Secondary 32F05, 31C10.

${ }^{1}$ Research partially supported by National Science Foundation Grants GP-27576 and GP-34785. The second author is a Sloan Fellow and was an SRC fellow at the University of Warwick when the bulk of this work was done.
} 
present Theorem 3 completely supersedes Theorem 6 of [1]. In case $M$ is an open subset of $\boldsymbol{C}^{n}$, we can prove that $M$ is a Stein manifold under otherwise much weaker hypotheses.

THEOREM 4. If $\boldsymbol{M}$ is an open subset of $\boldsymbol{C}^{n}$ which admits a complete Kähler metric $G$ of nonnegative sectional curvature, then every convex (relative to $G$ ) open subset of $M$ is a domain of holomorphy.

This theorem is an improvement of Corollary (A) of Theorem 3 in [2]. The next result complements Theorem 5 of [2]. We shall use the notation of that theorem plus the following: Given a complete Kähler manifold $M$ and a bounded subset $D$ of $M$, we let $r_{D}$ be the minimum of the Ricci curvature of $M$ in $\bar{D}$.

THEOREM 5. Let $M$ be as in Theorem 3. Let $D$ be a bounded pseudoconvex open subset in $M$ and let $\varphi$ be a plurisubharmonic function in $D$. Then, for any $f \in L_{(0, q)}^{2}(D, \varphi), q>0$, with $\bar{f} f=0$, we can find $u \in L_{(0, q-1)}^{2}(D, \varphi)$ such that $\bar{\partial} u=f$ and

$$
q r_{D} \int_{D}|u|^{2} e^{-\varphi} \Omega \leqq \int_{D}|f|^{2} e^{-\varphi} \Omega .
$$

The next theorem generalizes to certain Kähler manifolds of nonnegative curvature the fact that no nonzero holomorphic function on $C^{n}$ is in $L^{p}$. The theorem is a consequence of the following result concerning Riemannian manifolds: If $M$ is a complete noncompact Riemannian manifold of nonnegative sectional curvature and if $f \not \equiv 0$ is a nonnegative $C^{\infty}$ subharmonic function, then $\int_{M} f \Omega=+\infty$. (Here $\Omega=$ the Riemannian volume form on $M$ and $f$ being subharmonic means $\Delta f \geqq 0$ everywhere on $M$.)

THEOREM 6. Let $M$ be a complete noncompact Kähler manifold with nonnegative sectional curvature. Then no nonzero holomorphic function on $M$ is in $L^{p}$ for any $p$ satisfying $1 \leqq p<+\infty$.

We would like to propose another conjecture. In its most conservative form, it reads: A complete Kähler manifold with positive Ricci curvature and nonnegative sectional curvature is holomorphically convex. The following theorem should be useful in resolving this conjecture. Recall that an open subset $U$ of a complex manifold $M$ is said to be Runge in $M$ if given a holomorphic function $f$ on $U$ and a compact set $K \subseteq U$, there exists a holomorphic function $F$ on $M$ which approximates $f$ on $K$ arbitrarily closely. Also recall that a function on a Riemannian manifold is convex if its restriction to every geodesic is a convex function of one variable; a convex function is always continuous. 
THEOREM 7. Let $M$ be a complete noncompact Kähler manifold with positive Ricci curvature and nonnegative sectional curvature. Let $\varphi: M \rightarrow \boldsymbol{R}$ be a convex function such that each sublevel set $M_{c}=\{p \in M: \varphi(p)<c\}$ has compact closure in $M$. Then $M_{c}$ is Runge in $M$ for all $c \in \boldsymbol{R}$.

In closing, we remark that all the preceding theorems make essential use of the approximation theorem of Greene-Wu [3]; this fact is not surprising since that approximation theorem was proved with these applications in mind.

\section{REFERENCES}

1. R. E. Greene and $\mathrm{H}$. Wu, Curvature and complex analysis, Bull. Amer. Math. Soc. 77 (1971), 1045-1049. MR 44 \#473.

2. - Curvature and complex analysis. II, Bull. Amer. Math. Soc. 78 (1972), 866-870.

3. - On the subharmonicity and plurisubharmonicity of geodesically convex functions, Indiana Univ. Math. J. (to appear).

4. K. Kodaira, On Kähler varieties of restricted type (an intrinsic characterization of algebraic varieties), Ann. of Math. (2) 60 (1954), 28-48. MR 16, 952.

5. R. Remmert, Holomorphe und meromorphe Abbildungen komplexer Raüme, Math. Ann. 133 (1957), 328-370. MR 19, 1193.

Department of Mathematics, University of California, los Angeles, California 90024

Department of Mathematics, University of California, Berkeley, California 94720 